\title{
Coherent backscattering of light in a magnetic field
}

\author{
D. Lacoste and B. A. van Tiggelen \\ Laboratoire de Physique et Modélisation des Milieux Condensés, \\ CNRS, Universit Joseph Fourier, Maison des Magistères, \\ B.P. 166, 38042 Grenoble Cedex 09, France
}

October 23, 2018

\begin{abstract}
This paper describes how coherent backscattering is altered by an external magnetic field. In the theory presented, magnetooptical effects occur inside Mie scatterers embedded in a non-magnetic medium. Unlike previous theories based on point-like scatterers, the decrease of coherent backscattering is obtained in leading order of the magnetic field using rigorous Mie theory. This decrease is strongly enhanced in the proximity of resonances, which cause the path length of the wave inside a scatterer to be increased. Also presented is a novel analysis of the shape of the backscattering cone in a magnetic field.
\end{abstract}

\section{Introduction}

The enhancement of backscattering in propagation of waves in a random medium is a well documented topic. Weak localization theory explains how interference effects between direct and reverse scattering events produce Coherent Backscattering (CBS). The main features of CBS are insensitive to many aspects of the statistics of the inhomogeneities. Even absorption does not alter the relative strength of the interference [1]. The interference is only affected by the properties of the medium with respect to the reciprocity principle as first noted by Golubentsev [2]. MacKintosh and John [3] analyzed how CBS is altered by Faraday rotation Znd natural optical activity in a medium of inhomogeneities smaller than the wavelength, and in the diffusion approximation. U'sing a method based on point-like scatterers, Van Tiggelen et al. extended these ideas and discussed the anisotropy induced by a magnetic field in light diffusion [4. The case of non-magnetic Mie scatterers embedded in a Faraday-active medium has been studied by means of Monte Carlo simulations by Martinez et al. [5. Maradudin et al. considered specifically the two dimensional doherent backscattering of light from a randomly rough surface in the presence of a magnetic field [6]. Experimentally, CBS in a magnetic field has been studied by Erbacher, Lenke and Maret [7], and some of their results will be discussed here.

In section 2, the main results of a recent calculation of the T-matrix for a Mie scatterer in a magnetic field are presented 8. 9], and serve in section 3 as the building block to study diffusion of light in a magnetic field. After having detailed the main features of the Faraday effect for multiple Rayleigh and Mie scattering, the modification of the line shape of CBS in a medium Jith finite-size scatterers in a magnetic field is investigated in the last section.

\section{T-matrix in a magnetic field}

In this paper, $c_{0}=1$ has been set. In a magnetic field, the refractive index is a tensor of rank two. For the standard Mie problem, it depends on the distance to the center of the sphere $r$, which has a radius $a$, via the Heaviside function $\Theta(r-a)$, that Equals 1 inside the sphere and 0 outside,

$$
\varepsilon(\mathbf{B}, \mathbf{r})-\mathbf{I}=\left[\left(\varepsilon_{0}-1\right) \mathbf{I}+\varepsilon_{F} \Phi\right] \Theta(r-a) .
$$

In this expression, $\varepsilon_{0}=m^{2}$ is the value of the normal isotropic dielectric constant of the sphere of relative index of refraction $m$, and

$$
\varepsilon_{F}=2 m V_{0} B / \omega
$$

is a dimensionless coupling parameter associated with the amplitude of the Faraday effect ( $V_{0}$ being the Verdet constant, $B$ the amplitude of the magnetic field and $\omega$ the frequency). The antisymmetric hermitian tensor $\Phi_{i j}=i \epsilon_{i j k} \hat{B}_{k}$ has been introduced (the hat above vectors means that the vectors have been normalized). The Mie solution depends on the dimensionless size 
parameters $x=\omega a$ and $y=m x$. In this paper, only non-absorbing media are considered so that $m$ and $\varepsilon_{F}$ are real-valued. Since $\varepsilon_{F} \approx 10^{-4}$ in most experiments, a perturbational approach is valid. The part of $\mathbf{T}$ that is independent of the magnetic field is denoted $\mathbf{T}^{0}$, the part of the T-matrix linear in $\mathbf{B}$ is $\mathbf{T}^{1}$, and the second order correction $\mathbf{T}^{2}$.

Two important symmetry relations must be obeyed by a T-matrix of a scatterer in a magnetic field. The first one is parity symmetry and the second one is reciprocity [8]:

$$
\begin{gathered}
T_{-\mathbf{k} \sigma,-\mathbf{k}^{\prime} \sigma^{\prime}}(\mathbf{B})=T_{\mathbf{k} \sigma, \mathbf{k}^{\prime} \sigma^{\prime}}(\mathbf{B}), \\
T_{-\mathbf{k}^{\prime}-\sigma^{\prime},-\mathbf{k}-\sigma}(-\mathbf{B})=T_{\mathbf{k} \sigma, \mathbf{k}^{\prime} \sigma^{\prime}}(\mathbf{B}) .
\end{gathered}
$$

It is important to note that $\sigma(-\hat{\mathbf{k}})=-\sigma(\hat{\mathbf{k}})$, i.e. $\sigma$ indicates the helicity. In particular, relations (3, 国) must hold for $\mathbf{T}^{1}$. $\mathbf{T}^{2}$ satisfies Eq. (四) without a minus sign for $\mathbf{B}$ and obeys the standard reciprocity principle. For this reason, $\mathbf{T}^{2}$ will not contribute to the suppression of the backscattering cone considered in this article, the next order being $\mathbf{T}^{3}$.

Because $\mathbf{T}^{1}$ is linear in $\hat{\mathbf{B}}$, it can be constructed by considering only three special cases for the direction of $\hat{\mathbf{B}}$. If $\hat{\mathbf{k}}$ and $\hat{\mathbf{k}}^{\prime}$ are not collinear, the unit vector $\hat{\mathbf{B}}$ can be decomposed in the non-orthogonal but complete basis of $\hat{\mathbf{k}}, \hat{\mathbf{k}}^{\prime}$ and $\hat{\mathbf{g}}=\hat{\mathbf{k}} \times \hat{\mathbf{k}}^{\prime} /\left|\hat{\mathbf{k}} \times \hat{\mathbf{k}}^{\prime}\right|$. This results in [8],

$$
\begin{aligned}
\mathbf{T}_{\mathbf{k k}^{\prime}}^{1} & =\frac{(\hat{\mathbf{B}} \cdot \hat{\mathbf{k}})\left(\hat{\mathbf{k}} \cdot \hat{\mathbf{k}}^{\prime}\right)-\hat{\mathbf{B}} \cdot \hat{\mathbf{k}}^{\prime}}{\left(\hat{\mathbf{k}} \cdot \mathbf{k}^{\prime}\right)^{2}-1} \mathbf{T}_{\mathbf{k k}^{\prime}}^{1}\left(\hat{\mathbf{B}}=\hat{\mathbf{k}}^{\prime}\right)+\frac{\left(\hat{\mathbf{B}} \cdot \hat{\mathbf{k}}^{\prime}\right)\left(\hat{\mathbf{k}} \cdot \hat{\mathbf{k}}^{\prime}\right)-\hat{\mathbf{B}} \cdot \hat{\mathbf{k}}}{\left(\hat{\mathbf{k}} \cdot \hat{\mathbf{k}}^{\prime}\right)^{2}-1} \mathbf{T}_{\mathbf{k} \mathbf{k}^{\prime}}^{1}(\hat{\mathbf{B}}=\hat{\mathbf{k}}) \\
& +(\hat{\mathbf{B}} \cdot \hat{\mathbf{g}}) \mathbf{T}_{\mathbf{k k}^{\prime}}^{1}(\hat{\mathbf{B}}=\hat{\mathbf{g}}),
\end{aligned}
$$

With respect to an helicity base, $\mathbf{T}^{1}$ takes the form,

$$
\begin{aligned}
T_{\sigma \sigma^{\prime}}^{1}(\hat{\mathbf{B}}=\hat{\mathbf{k}}) & =\frac{\pi}{\omega}\left[R_{1}(\theta) \sigma+R_{2}(\theta) \sigma^{\prime}\right], \\
T_{\sigma \sigma^{\prime}}^{1}\left(\hat{\mathbf{B}}=\hat{\mathbf{k}}^{\prime}\right) & =\frac{\pi}{\omega}\left[R_{1}(\theta) \sigma^{\prime}+R_{2}(\theta) \sigma\right],
\end{aligned}
$$

with

$$
\begin{aligned}
& R_{1}(\theta)=-\frac{2 \varepsilon_{F}}{m} \sum_{J \geq 1} \frac{2 J+1}{J(J+1)}\left[\mathcal{C}_{J} \pi_{J, 1}(\theta)+\mathcal{D}_{J} \tau_{J, 1}(\theta)\right] \\
& R_{2}(\theta)=-\frac{2 \varepsilon_{F}}{m} \sum_{J \geq 1} \frac{2 J+1}{J(J+1)}\left[D_{J} \pi_{J, 1}(\theta)+C_{J} \tau_{J, 1}(\theta)\right]
\end{aligned}
$$

where the coefficients $\mathcal{C}_{J}$ and $\mathcal{D}_{J}$ have been defined in Ref. [8]. For $\mathbf{T}_{\sigma \sigma^{\prime}}^{1}(\hat{\mathbf{B}}=\hat{\mathbf{g}})$, the following expression has been found

$$
T_{\sigma \sigma^{\prime}}^{1}(\hat{\mathbf{B}}=\hat{\mathbf{g}})=\frac{\pi}{\omega}\left(Q_{1}+\sigma \sigma^{\prime} Q_{2}\right)
$$

with

$$
Q_{1}=-i \frac{d}{d \theta} R_{1}, \quad Q_{2}=-i \frac{d}{d \theta} R_{2}
$$

For $\hat{\mathbf{k}}=\hat{\mathbf{k}}^{\prime}$,

$$
\mathbf{T}_{\mathbf{k}, \mathbf{k}}^{1}=\Phi \frac{2 \pi}{\omega} R_{1}(0)
$$

with

$$
R_{1}(0)=R_{2}(0)=-\frac{\varepsilon_{F}}{m} \sum_{J \geq 1}(2 J+1)\left(\mathcal{C}_{J}+\mathcal{D}_{J}\right) .
$$




\section{Diffusion of light in a magnetic field}

The equation of radiative transfer aims to describe the propagation of the average intensity in multiple scattering, but violates reciprocity [10]. The reason is that it does not contain the most-crossed diagrams $\left(C_{i j k l}^{+}\right)$responsible for CBS. By definition, the four-rank tensor $L_{i j k l}$ linearly connects the field correlations of incident and outgoing fields. This can be represented by a diagram that starts on the first scattering event, and which has two lines, corresponding to the propagation of the field and to the complex conjugate of the field as can be seen in Fig. (1). For $L_{i j k l}^{+}$both fields propagate in the same magnetic field $\mathbf{B}$ as opposed to $L_{i j k l}^{-}$for which both fields propagate with opposite sign for the magnetic field $\mathbf{B}$ 团. The relations deduced from the application of the reciprocity relation (4) are

$$
C_{i j l k}^{ \pm}\left(\mathbf{p}, \mathbf{p}^{\prime}, \mathbf{q}, \mathbf{B}\right)=L_{i j k l}^{\mp}\left(\frac{\mathbf{p}-\mathbf{p}^{\prime}+\mathbf{q}}{2}, \frac{\mathbf{p}^{\prime}-\mathbf{p}+\mathbf{q}}{2}, \mathbf{p}+\mathbf{p}^{\prime}, \mathbf{B}\right)
$$

and

$$
L_{i j k l}^{ \pm}\left(\mathbf{p}, \mathbf{p}^{\prime}, \mathbf{q}, \mathbf{B}\right)=L_{j i l k}^{ \pm}\left(-\mathbf{p}^{\prime},-\mathbf{p},-\mathbf{q},-\mathbf{B}\right) .
$$

On long length scales $(\mathbf{q} \rightarrow 0)$ and for stationary situations $(\Omega=0)$, the ladder diagrams $L_{i j k l}^{-}$take the following hydrodynamic form [11],

$$
L_{i j k l}^{-}\left(\mathbf{p}, \mathbf{p}^{\prime}, \mathbf{q}, \mathbf{B}\right)=\frac{2 \pi}{\ell^{2}} \frac{d_{i k}(\mathbf{p}, \mathbf{q}, \mathbf{B}) d_{l j}\left(-\mathbf{p}^{\prime},-\mathbf{q},-\mathbf{B}\right)}{D \mathbf{q}^{2}+\lambda / \ell} .
$$

The symmetry of the numerator is imposed by the reciprocity principle; $\lambda$ is a scalar dimensionless parameter that elucidates the breaking of reciprocity by the magnetic field in multiple scattering. In Eq. (16), $D$ is the conventional diffusion coefficient for radiative transfer, and $\ell$ is the scattering mean free path, which is a typical distance between two scattering events. This diffusion constant was shown theoretically and experimentally [12] to depend on the square of the magnetic field. These corrections are not considered here since the dephasing parameter $\lambda /(D \ell)$ is only discussed to second order in the magnetic field. The enhancement factor of coherent backscattering is a ratio of a coherent contribution, described by the most-crossed diagrams, over an incoherent contribution represented by the ladder diagrams. The parameter $\lambda$ plays the role of an "absorption" term for the coherent contribution only, and is therefore responsible for the decrease of the coherent backscattering cone in a magnetic field. This parameter $\lambda$ can be expressed as the square of a Faraday dephasing angle, which is the product of a Verdet constant, the magnetic field strength, and a characteristic length scale for the propagation of light in the medium. Since the scattering mean free path $\ell$ is a natural and experimentally relevant length scale for coherent backscattering, it is possible to define an effective Verdet constant $V_{\text {eff }}$ from a relation derived in [3],

$$
\lambda=\frac{4}{3} V_{e f f}^{2} B^{2} \ell^{2} .
$$

Equation (17) was obtained by MacKintosh and John [3], who considered a situation where the scatterers and the outside medium both have a Verdet constant $V_{\text {eff }}$.

To first order in the magnetic field, the tensor $d_{i k}$ defined in Eq. (16) must take the form

$$
\mathbf{d}(\mathbf{p}, \mathbf{q}=0, \mathbf{B})=\mathbf{I}+d_{2} \Phi .
$$

where $d_{2}$ describes persisting polarization effects in diffuse scattering due to the magnetic field [11]. For low density $n$ of particles, $d_{2}$ can be determined from the Bethe-Salpether equation

$$
L_{\mathbf{p} \mathbf{p}^{\prime}}^{-}(\mathbf{B})=n \mathbf{T}_{\mathbf{p} \mathbf{p}^{\prime}}(\mathbf{B}) \mathbf{T}_{\mathbf{p} \mathbf{p}^{\prime}}^{*}(-\mathbf{B})+n \sum_{\mathbf{p}^{\prime \prime}} \mathbf{T}_{\mathbf{p p}^{\prime \prime}}(\mathbf{B}) \cdot \mathbf{G}\left(\mathbf{p}^{\prime \prime}, \mathbf{B}\right) \cdot \mathbf{G}^{*}\left(\mathbf{p}^{\prime \prime},-\mathbf{B}\right) \cdot \mathbf{T}_{\mathbf{p} \mathbf{p}^{\prime \prime}}^{*}(-\mathbf{B}) \cdot L_{\mathbf{p}^{\prime \prime} \mathbf{p}^{\prime}}^{-}(\mathbf{B}),
$$

where $\mathbf{G}(\mathbf{p}, \mathbf{B})$ denotes the ensemble averaged Dyson Green's tensor (see Appendix A for details of notations), and the asterisk denotes hermitian conjugation in polarization space. This equation is slightly more complicated than the one for $L_{\mathbf{p p}^{\prime}}^{+} 11$ because it involves both $\mathbf{B}$ and $\mathbf{- B}$ due to the definition (16). Inserting Eqs. (16) and (18) into Eq. (19) and expanding to first and second order in the magnetic field using Eq. (5), fixes $d_{2}$ and $\lambda$ rigorously. The final result reads

$$
d_{2}=i\left(\frac{-A_{2}}{1-\langle\cos \theta\rangle_{p}}-\eta\right)
$$




$$
\lambda=\frac{1}{3}\left(A_{1}^{2}+\frac{A_{2}^{2}}{1-\langle\cos \theta\rangle_{p}}\right),
$$

with

$$
\begin{gathered}
A_{1}^{2}=\frac{1}{x^{2} Q_{\text {scatt }}} \int_{-1}^{1} d \mu\left[2 \frac{\left|R_{1}\right|^{2}+\left|R_{2}\right|^{2}-2 \Re e\left(R_{1} \overline{R_{2}}\right) \mu}{1-\mu^{2}}+\left|Q_{1}\right|^{2}+\left|Q_{2}\right|^{2}\right], \\
A_{2}=\frac{1}{x^{2} Q_{\text {scatt }}} \int_{-1}^{1} \Re e\left(R_{1} S_{2}+R_{2} S_{1}\right) d \mu-\eta .
\end{gathered}
$$

Equations (20 23) contain the main results of this paper and will be discussed in sections 5 to 8 . The factor $1 /\left(1-\langle\cos \theta\rangle_{p}\right)$ in Eqs. (20) and (21) is related to a depolarization length which will be introduced in the next section. In equation (21), $\lambda$ contains two terms: the first one is seen to dominate for finite size particles $(x \gg 1)$ whereas the second one prevails for small particles $(x \ll 1)$. Sections 5 and 6 will discuss these two contributions respectively. Far from resonances, the first term corresponds to the Faraday rotation of the wave inside the scatterer, whereas the second represents the Faraday rotation between two scattering events. Although the medium outside the scatterers is not Faraday active, the Faraday rotation from one scatterer to the next can be defined in the framework of an effective medium theory using the parameter $\eta$, which will be defined in section 0 .

\section{Depolarization length}

In this section, the factor $1 /\left(1-\langle\cos \theta\rangle_{p}\right)$, which tends to amplify the second term of Eq. (21) is defined. The term $A_{2}^{2}$ is easily seen to dominate for small particles. The total cross-section of one particle is given by [13],

$$
Q_{\text {scatt }}=\frac{1}{x^{2}} \int_{-1}^{1}\left(\left[\left.S_{1}\right|^{2}+\left|S_{2}\right|^{2}\right) d \mu\right.
$$

with $\mu=\cos \theta$. The transport mean free path is defined as $\ell^{*}=\ell /(1-\langle\cos \theta\rangle)$, where the asymmetry parameter $\langle\cos \theta\rangle$ is given by

$$
\langle\cos \theta\rangle=\frac{1}{x^{2} Q_{\text {scatt }}} \int_{-1}^{1}\left(\left[\left.S_{1}\right|^{2}+\left|S_{2}\right|^{2}\right) \mu d \mu .\right.
$$

Likewise, a depolarization length can be defined as $\ell_{\text {dep }}=\ell /\left(1-\langle\cos \theta\rangle_{p}\right)$, where

$$
\langle\cos \theta\rangle_{p}=\frac{1}{x^{2} Q_{\text {scatt }}} \int_{-1}^{1} 2 \Re e\left(S_{1} \overline{S_{2}}\right) \mu d \mu
$$

Rayleigh scattering has a forward-backward symmetry so that $\langle\cos \theta\rangle=0$. However, scattering is not isotropic due to the polarization and one can easily show that for Rayleigh scatterers $\langle\cos \theta\rangle_{p}=0.5$. In the limit of large forward scattering and for $m=1.33$, both asymmetry parameters, $\langle\cos \theta\rangle$ and $\langle\cos \theta\rangle_{p}$ tend towards a limit close to 0.85 , as shown in Fig. (22a). In the forward direction the differences between the two states of polarization vanish since $S_{1}(0)=S_{2}(0)$.

The well-known oscillations and ripple structure 13 of the asymmetry parameter $\langle\cos \theta\rangle$ are also present in $\langle\cos \theta\rangle_{p}$. As shown in Fig. (2b), for a relative high value of the relative index of refraction $\left(m=2.73\right.$ corresponding to $\left.\mathrm{TiO}_{2}\right)$, the asymmetry parameters $\langle\cos \theta\rangle$ and $\langle\cos \theta\rangle_{p}$ may take negative values, which can be seen in this particular case near $x=2$. In this very particular case, where the scattering is essentially in the backward direction, the characteristic length for the loss of the polarization $\ell_{\text {dep }}$ can be smaller than the characteristic length for the loss of the phase in multiple light scattering, which is $\ell$.

\section{$5 \quad$ Faraday rotation for multiple Rayleigh scattering}

Equation (20) for $d_{2}$ and the second term in Eq. (21) can be understood from an "effective medium" theory, valid for Rayleigh scatterers. The real part of the forward scattering amplitude (12) is associated with the Faraday effect and the imaginary part with magneto-dichroism (i.e different absorption for different circular polarization) of an ensemble of Faraday-active scatterers. For a dilute system, the antisymmetric part of this effective refractive index $\varepsilon_{a}$ is defined as 


$$
\varepsilon_{a}=-\frac{2 \pi n}{\omega^{3}} R_{1}(0)
$$

For the real part of this effective refractive index, the dimensionless parameter $\eta$ is defined by

$$
\eta=\Re e\left(\varepsilon_{a}\right) k \ell
$$

Equations (20) and (23) involve this parameter $\eta$, which represents a characteristic phase in multiple scattering, due to the Faraday effect in the effective medium accumulated over a distance $\ell$. An ensemble of Rayleigh scatterers (for which the electromagnetic field changes only slightly on a scale comparable to the particle size) has the finite value

$$
\varepsilon_{a}=\frac{9 f \varepsilon_{F}}{\left(m^{2}+2\right)^{2}} .
$$

The Faraday effect of a composite material made of particles smaller than the wavelength, and of different shape (spherical, needle like, plate like) was discussed in Ref. [14], using a more general version of the effective medium approximation (not limited to dilute samples).

For Rayleigh scatterers $(x \rightarrow 0)$, one obtains

$$
\begin{gathered}
d_{2}=\frac{9 i \varepsilon_{F}}{4\left(m^{2}-1\right)^{2} x^{3}} \\
\lambda=\frac{27 \varepsilon_{F}^{2}}{8\left(m^{2}-1\right)^{4} x^{6}},
\end{gathered}
$$

so that, by definition (17),

$$
\frac{V_{e f f}}{f V_{0}}=\frac{9 \sqrt{2} m}{\left(m^{2}+2\right)^{2}} .
$$

Apart from a factor depending on the index of refraction, the effective Verdet constant is found to be the product of the volume fraction of the particles by their Verdet constant. As noticed before [4], one finds a factor of $\sqrt{2}$ more than expected on the basis of the effective medium approach of Eq. (27) if $V_{\text {eff }}$ would have defined by $\varepsilon_{a}=2 V_{\text {eff }} B / \omega$. This discrepancy is due to the denominator in Eq. 21], $1-\langle\cos \theta\rangle_{p}=1 / 2$ for Rayleigh particles.

From the experimental parameters described in the experiments of Erbacher et al. (a relative index of refraction of $m=1.15$ ), the estimate using Eq. (28) is

$$
\frac{V_{e f f}}{f V_{0}} \approx 1.32
$$

This value is to be compared with the experimental value of $1.55 \pm 0.15$ [7]. The proximity of the two values probably explains the success of previous theories based on Rayleigh scatterers, although this experiment dealt with Mie particles. In this experiment, the maximum of the distribution of the size parameters was roughly estimated at $x \simeq 23$ but the width of the distribution was very broad. Using the parameters of Erbacher et al., our Mie theory reproduces the limit of Eq. (29) for $x=0$, but predicts a value of only 0.4 at $x \simeq 23$. The solid line in figure 3 a represents the effective Verdet constant as a function of the size parameter $x$, for the same relative index of refraction used in the experiment of Erbacher et al.. One can clearly see in this figure that a distribution of large spheres of size parameter $x$ of the order of 20 or higher can not explain the experimental result. However if the size distribution of the scatterers was rather centered around a size parameter of roughly 10, the experimental value of the effective Verdet constant could possibly be recovered from this Mie theory. In figure $3 \mathrm{~b}$, an higher value of the relative index was chosen. In this case, the effective Verdet constant is seen to be enhanced by resonances, which will be the subject of the section 7 .

\section{Faraday rotation for multiple Mie scattering}

The first term in Eq. 21]) originates from the Faraday effect inside the scatterers and is the main contribution in the Mie regime of $x \gg 1$. Resonances will be discussed in section 7. Using the definition of Eq. (17), the effective Verdet constant of Mie particles is written as 


$$
\frac{V_{\text {eff }}}{f V_{0}}=\frac{3 m Q_{\text {scatt }} \sqrt{3 \lambda}}{4 x \varepsilon_{F}} .
$$

When the size parameter obeys $x \gg 1$ and $y=m x \gg 1$, the scattering can be interpreted in terms of geometrical optics. In geometrical optics, rays incident on the sphere are considered rather than plane waves. The ray with central impact is characterized in Mie theory by $J=1$. In figure 3 a, the effective Verdet constant, that contains contributions from all the rays, is plotted as a solid line, with respect to the size parameter $x$. The dashed line in this figure represents the separated contribution of the ray with central impact $J=1$ in the effective Verdet constant. The two curves merge for $x=0$, the Rayleigh limit, but deviate from each other for larger size parameter. For a given value of the size parameter, it can be noted that the ray with central impact already represents a significant contribution to the effective Verdet constant. In addition to this, the effective Verdet constant is seen to decrease for increasing size parameter $x$. This is an important observation, because this means that Mie scatterers of large size (typically $x>20$ ) are less efficient than Rayleigh scatterers in suppressing the coherent backscattering cone.

\section{Mie resonances}

Let us first recall some results for resonant Rayleigh scattering, for which the resonance behavior is analogous to the resonance of a two-level atom in atomic physics. For resonant Rayleigh scatterers the effective Verdet constant is related to the "path length" $L_{\text {path }}$ of the wave inside the particle. In fact, the path length, the dwell time of the light and the total electromagnetic energy stored inside the scatterer have been seen to be proportional. The path length can be defined as (see formula (2-22) P17 of Ref. 15]),

$$
L_{\text {path }}=\lim _{m_{i} \rightarrow 0} \frac{Q_{a b s}}{\omega m_{i}},
$$

where $Q_{a b s}$ is the absorption cross-section and $m_{i}$ the imaginary part of the index of refraction. The physical idea behind this definition is that the longer the path of the light is in the particle, the more the light will suffer from absorption. For resonant Rayleigh particles, the relation to the effective Verdet constant is obtained from Eqs. (28.31),

$$
\frac{V_{e f f}}{f V_{0}}=\frac{4 m_{r} \sqrt{2}}{3} \frac{L_{p a t h}}{a} .
$$

At resonance, the path length can exceed the size of the scatterer, which means that the effective Verdet constant should be strongly enhanced by resonant scattering. Alternatively, one may relate the path length to the time spent by the wave in the medium, which means that the Faraday rotation is in some sense a Larmor clock, measuring this time [16].

The question is whether resonant enhancement of Faraday rotation occurs in resonant Mie scattering. For Mie resonances the increase in path length is well related to the change of the electromagnetic energy within the scatterers with respect to the surrounding. The total time-averaged electromagnetic energy inside the sphere is denoted by $W$ and $W_{0}$ represents this energy for the incident plane wave. For weak absorption, $m_{i} \ll m_{r}$, the electromagnetic energy $W$ can be approximated by [17]

$$
\frac{W}{W_{0}} \simeq \frac{3 m_{r} Q_{a b s}}{8 x m_{i}}=\frac{3 m_{r} L_{p a t h}}{a} .
$$

where Eq. (31) was applied to obtain the last equality. This relation is exact for scalar waves and is a very good approximation for vector waves. It is even an excellent approximation in the vicinity of resonances were the deviations between the exact solution and its approximation are the largest. In the domain of $J \simeq x$, several resonances take place in the Mie coefficients $a_{J}$ and $b_{J}$. These resonances are well separated and can be numbered by an additional integer $k$ the order of the resonance [18]. Near one electric Mie resonance of a specified order, the denominators of the Mie coefficients $a_{J}$ and $c_{J}$, which are identical, are close to zero. From Eqs. (21]22), one finds $\sqrt{\lambda} \sim\left|c_{J}\right|^{2} /\left|a_{J}\right|$. By Eqs. (30) and (33), this implies

$$
\frac{V_{e f f}}{f V_{0}} \sim\left|c_{J}\right|^{2}\left|a_{J}\right| \sim \frac{W}{W_{0}}\left|a_{J}\right| \sim \frac{W}{W_{0}} .
$$

In the last equality the role of $\left|a_{J}\right|$ is dominated by $\left|c_{J}\right|^{2}$ at resonance, since resonances in the scattering cross-section $Q_{\text {scatt }}$ are much less significant than resonances in $W$. Indeed, in Fig. (耳a), the lower curve represents $Q_{\text {scatt }}$, on normal scale, near one resonance of a water sphere. It is much below the curves of $W$ or $V_{\text {eff }} /\left(f V_{0}\right)$ which are even plotted on a logarithmic scale. From 
Eq. (34), the effective Verdet constant is expected to be simply proportional to the electromagnetic energy $W$ (or equivalently to $L_{\text {path }}$ ). This generalizes the result for Rayleigh scatterers in Eq. (32). The numerical verification of Eq. (34) can be deduced from the double logarithmic plot of $V_{\text {eff }} /\left(f V_{0}\right)$ against the total electromagnetic energy $W$ in Fig. (佔).

The proportionality of the Verdet constant and the total electromagnetic energy (or equivalently $L_{\text {path }}$ ) has been derived for the particular case where the path of the light is confined along the same line (1D problem as in a Fabry-Perot configuration for instance [8]). In this case, the cumulative character of the Faraday rotation with respect to the path length leads to an experimentally observed enhancement of the Faraday rotation [19]. Eq. (34) applies to any resonant impact and shows that the possible occurrence of spin flips in Mie scattering, as suggested in Ref. [7, does not affect the behavior of the effective Verdet constant near resonances and that the Faraday rotation still accumulates along the path as in the 1D case. This is consistent with the observation made in section 6 , that the ray with central impact had an important role for interpreting the Faraday rotation for multiple Mie scattering.

In conclusion, like for resonant Rayleigh particles, a strong correlation between the effective Verdet constant and the stored energy inside the sphere was found for resonant Mie particles. The general behavior and proportionality in the vicinity of a resonance is apparently universal.

\section{Shift of the intensity profile of the coherent backscattering cone in a mag- netic field}

A magnetic field can be expected to introduce some anisotropy in the light intensity profile of the cone. In this section, the form taken by this anisotropy is investigated by taking care of the selection rules imposed for the polarization in reflection of a semi-infinite system of Mie scatterers. This analysis is restricted to linear corrections in the magnetic field, so that the parameter $\lambda$ discussed in the former section, quadratic in the field, will no longer appear.

This approach is based on an improved version of the scalar diffusion approximation. The ladder propagator at point $\mathbf{r}=\left\{\mathbf{r}_{\perp}, z\right\}$ for a source at $\mathbf{r}^{\prime}=\left\{\mathbf{r}_{\perp}^{\prime}, z^{\prime}\right\}$ in a semi-infinite medium is denoted by $\rho\left(\mathbf{r}, \mathbf{r}^{\prime}\right)$. The $z$-axis is directed along the normal of the sample, and $\mathbf{r}_{\perp}$ and $\mathbf{r}_{\perp}^{\prime}$ are vectors perpendicular to the $z$-axis. Because of translational invariance in the plane of the sample, the ladder propagator only depends on $\mathbf{r}_{\perp}-\mathbf{r}_{\perp}^{\prime}$. The two-dimensional Fourier transform of $\rho\left(\mathbf{r}, \mathbf{r}^{\prime}\right)$ with respect to $\mathbf{r}_{\perp}-\mathbf{r}_{\perp}^{\prime}$ is denoted by $\widetilde{\rho}\left(\mathbf{q}, z, z^{\prime}\right)$. The ladder propagator $\rho\left(\mathbf{r}, \mathbf{r}^{\prime}\right)$ obeys the following diffusion equation

$$
\left(-\nabla^{2}+\frac{1}{L_{a}^{2}}\right) \rho\left(\mathbf{r}, \mathbf{r}^{\prime}\right)=\delta\left(\mathbf{r}-\mathbf{r}^{\prime}\right)
$$

with the radiative boundary condition [20]

$$
\forall \mathbf{r}_{\perp}, \forall \mathbf{r}_{\perp}^{\prime}, \forall z>0, \quad \rho\left(\left\{\mathbf{r}_{\perp}, z\right\},\left\{\mathbf{r}_{\perp}^{\prime}, z^{\prime}=-z_{0}\right\}\right)=0 .
$$

The trapping plane is located at a distance $z_{0}=2 \ell^{*} / 3$ outside the sample, and $L_{a}$ is the absorption length for the light intensity. We assume that the first and the last scattering events take place one transport mean free path $\ell^{*}$ away from the boundary, in the directions specified by the incoming wavevector $\mathbf{p}$ and the outgoing wavevector $\mathbf{p}^{\prime}$. This allows to calculate the contribution of the ladder diagrams $L_{i j k l}^{+}\left(\mathbf{p}, \mathbf{p}^{\prime}, \mathbf{q}, \mathbf{B}\right)$, the so-called incoherent contribution to the coherent backscattering, directly from $\widetilde{\rho}\left(\mathbf{q}=0, z-\ell^{*} \hat{p}_{z}, z^{\prime}+\ell^{*} \hat{p}_{z}^{\prime}\right)$. Similarly, the coherent contribution is obtained from the most-crossed diagrams $C_{i j l k}^{+}\left(\mathbf{p}, \mathbf{p}^{\prime}, \mathbf{q}, \mathbf{B}\right)$, which are derived from $L_{i j k l}^{-}\left(\mathbf{p}, \mathbf{p}^{\prime}, \mathbf{q}, \mathbf{B}\right)$ using Eq. (14). These derivations are detailed out in Appendix A. The coherent contribution in an helicity basis and at reflection $\left(z=0, z^{\prime}=0\right)$ reads,

$$
C_{\sigma \sigma^{\prime}}^{+}=\widetilde{\rho}\left(\mathbf{p}_{\perp}+\mathbf{p}_{\perp}^{\prime},-\ell^{*} \hat{p}_{z}, \ell^{*} \hat{p}_{z}^{\prime}\right) \times \delta_{\sigma \sigma^{\prime}} \times\left(1+2 b_{1} \omega \ell \operatorname{det}\left(\hat{\mathbf{p}}_{\perp}^{\prime}, \hat{\mathbf{p}}_{\perp}, \hat{\mathbf{B}}\right)-2 \sigma b_{2} \omega \ell \hat{\mathbf{B}} \cdot\left(\hat{\mathbf{p}}_{\perp}+\hat{\mathbf{p}}_{\perp}^{\prime}\right)\right) .
$$

where the Kronecker symbol $\delta_{\sigma \sigma^{\prime}}$ guarantees conservation of helicity $\sigma(\hat{\mathbf{p}})=\sigma^{\prime}\left(\hat{\mathbf{p}}^{\prime}\right)$, and $b_{1}$ and $b_{2}$ are real-valued coefficients to be determined. Most experiments on coherent backscattering were done in the helicity-conserving channel, which has the advantage of having a maximal enhancement factor (since the contribution from single scattering vanishes in this case) and of having an isotropic line shape. Eq. (37) states that the magnetic field modifies the cone exactly in this channel, in agreement with previous work [3]. Even when a magnetic field is present, no coherent backscattering is found in the opposite-helicity channel, at least according to the present diffusion approximation.

Only the components of the magnetic field along the slab contribute in the r.h.s of Eq. (37). When the field is perpendicular to the slab, the decrease of the enhancement factor, described by $\lambda$, is the sole impact of the magnetic field and is not included in the present approximation. When the magnetic field is in the plane of the slab, two corrections show up. The first one, 
proportional to $b_{1}$, is magneto-transverse, since it produces a shift of the intensity profile of the cone in the plane of the slab, normal to the magnetic field. This correction is independent of the state of helicity of the light. The second correction in Eq. (37) proportional to $b_{2}$ does depend on the helicity $\sigma$. It produces a shift of the intensity profile of the cone in the direction of the magnetic field in the plane of the slab, quite similar to the correction induced by the magnetic field in the group velocity [21].

The coefficient $b_{1}$ can be calculated independently from $b_{2}$, in a way exactly analogous to the calculation of $a_{1}$ in Ref. 11 responsible for the Photonic Hall Effect (PHE). The result is

$$
b_{1}=\frac{1}{(1-\langle\cos \theta\rangle)^{2}} \frac{\int_{-1}^{1} d \cos \theta \sin \theta \sum_{\sigma \sigma^{\prime}} \Im m\left(T_{\sigma \sigma^{\prime}}^{0}(\theta) \overline{T_{\sigma \sigma^{\prime}}^{1}}(\hat{\mathbf{B}}=\hat{\mathbf{g}}, \theta)\right)}{2 \int_{-1}^{1} d \cos \theta \sum_{\sigma \sigma^{\prime}}\left|T_{\sigma \sigma^{\prime}}^{0}(\theta)\right|^{2}},
$$

where the magneto T-matrix $T_{\sigma \sigma^{\prime}}^{1}(\hat{\mathbf{B}}=\hat{\mathbf{g}})(\theta)$ was introduced in Eq. (10). Note that the imaginary part in Eq. (38) discriminates $b_{1}$ from the parameter $a_{1}$ responsible for the PHE, in which the real part figures. For Rayleigh scattering, one can readily prove that the parameters $b_{1}$ and $b_{2}$ both vanish. The calculation of $b_{2}$ for Mie scattering is very complicated and is beyond the scope of this paper.

The expected modification of the lines of equal enhancement factor due to the magnetic field is now investigated. Equation (37) translates into a CBS line shape

$$
E(\mu, \varphi)=1+C(\mu)\left(1+2 b_{1} \mu \sin \varphi-2 b_{2} \sigma \mu \cos \varphi\right) .
$$

The enhancement factor, in the helicity-conserving channel, is denoted by $E$ for a state of helicity $\sigma$. The dimensionless parameter $\mu=\omega \ell \theta$ and $C(\mu)$ the well-documented line shape of the cone without applied magnetic field were introduced [- The azimuthal angle between the projection of the outgoing wave vector into the plane of the slab and the magnetic field direction, which has been chosen along the $x$-axis, was denoted by $\varphi$. For simplicity, only the magneto-transverse correction proportional to $b_{1}$ will be considered here, so that $b_{2}=0$. This case corresponds to unpolarized incident light, for which the term proportional to $b_{2}$ in Eq. (39) vanishes. The pattern of the lines of equal enhancement factor associated with the $b_{2}$-correction alone is the same as the one of the transverse correction after a rotation of angle $\pi / 2$ about the $x$-axis. For a typical experiment [22] with $\mathrm{CeF}_{3}$ particles of approximate radius of $2 \mu \mathrm{m}$ at room temperature, with a wavelength $\lambda=457 \mathrm{~nm}$, Eq. (38) leads to the estimation $b_{1} \simeq 1.8 \cdot 10^{-2}$. The experimentally measured mean free path for a volume fraction of $f=0.1$ is $\ell^{*} \simeq 90 \mu \mathrm{m}$. Eq. (39) is valid for $b_{1} \mu \ll 1$, which means that our approach is limited to the angular domain $|\theta| \leq 0.8 \mathrm{rad}$. The equation for the lines of constant enhancement factor $E_{0}$ in the absence of magnetic field is $E_{0}=1+C\left(\mu^{0}\right)$, independent of the azimuthal angle $\varphi$. The first order correction in the magnetic field is separated by writing $\mu=\mu^{0}+\mu^{1}$. Equation (39) gives

$$
\mu^{1}=-2 b_{1} \frac{C\left(\mu^{0}\right)}{C^{\prime}\left(\mu^{0}\right)} \mu^{0} \sin \varphi .
$$

In Fig. (5), the polar diagram of the lines of constant enhancement factor is shown for $b_{1}=1.8 \cdot 10^{-2}$. As apparent from Eqs. (39,40), the distortion of the lines should increase away from backscattering (at exact backscattering there is no modification at all to first order in the magnetic field). As a consequence, with the value of $b_{1}$ given above only a modification of the line shape in the wings might be observed. Although the condition $b_{1} \mu \ll 1$ limits the domain of validity of the approximation there should nevertheless be a sufficiently broad angular range, in the wings of the cone, where the magnetic corrections could be visible. In figure 5 the condition of validity of the approximation has been satisfied.

Another condition of validity lies in the use of the diffusion approximation. This approximation predicts a $1 / \mu^{2}$ behavior for the line shape of the cone in the wings, which is actually a wrong result. In the wings, the contribution of lowest orders of scattering is dominant and not properly taken into account in the diffusion approximation. Using an exact theory, Gorodnichev proved that the outcome is a $1 / \mu$ dependence [23]. His result has been derived only for point-like scatterers but it should also be valid for Mie scatterers when the mean free path is much larger than the wavelength. In any case, the magnetic correction in Eq. (40) depends only on the logarithmic derivative of $C\left(\mu^{0}\right)$, which should change only by a factor of two if the actual law is $1 / \mu$ or $1 / \mu^{2}$, the general pattern of the lines of equal enhancement factor being not modified. Therefore, the shift of the center of mass of the light intensity profile which was calculated, should be fairly robust with respect to the exact form of $C\left(\mu^{0}\right)$.

\section{Conclusion}

This paper describes two modifications on the coherent backscattering cone produced by a magnetic field. The first one, the decrease of the enhancement factor, depends on the parameter $\lambda$ quadratic in the magnetic field, and was observed experimentally. 
The second modification is related to the anisotropy of the light intensity and appears already in linear order of the magnetic field. Preliminary experiments seem to have reported the possibility of a shift in the intensity profile [24]. Our analysis applies to spherical scatterers of any size that are Faraday active. The decrease of the backscattering cone gets less pronounced as the size of the scatterers increases, whereas the shift in the intensity profile is only possible with finite size scatterers. As was surmised in Ref. [7], the effective Verdet constant defined from the decrease of the cone is enhanced near Mie resonances. The effective Verdet constant is found to be intimately related to the stored electromagnetic energy i.e the dwell time of the light in the particle.

We acknowledge R. Lenke for making available his recent experimental work on the effect of a magnetic field on coherent backscattering light. We thank JJ. Greffet for stimulating discussions.

\section{A Derivation of the magnetic corrections to the tensor $C_{\sigma \sigma^{\prime}}^{+}$}

In this appendix, the notations are explained and a demonstration of Eq. (37) is given. The transverse part of the free Green tensor is denoted

$$
\mathbf{G}_{0}=\frac{\Delta_{\hat{\mathbf{p}}}}{\left(\omega / c_{0}\right)^{2}+i \varepsilon-p^{2}}
$$

with $\left(\Delta_{\hat{\mathbf{p}}}\right)_{i j}=\delta_{i j}-\hat{p}_{i} \hat{p}_{j}$ the projector upon the space orthogonal to $\mathbf{p}$. Similarly, the hermitian projector on the space orthogonal to $\mathbf{p}$ for a given state of helicity $\sigma$ is $P_{i j}^{\sigma}(\hat{\mathbf{p}})=\frac{1}{2}\left(\left(\Delta_{\hat{\mathbf{p}}}\right)_{i j}-i \sigma \varepsilon_{i j k} \hat{p}_{k}\right)$.

Generalizing Eq. (18) for finite value of $\mathbf{q}$ (to first order in $\mathbf{q}$ and in the magnetic field $\mathbf{B}$ ) gives

$$
\mathbf{d}(\mathbf{p}, \mathbf{q}, \mathbf{B})=\mathbf{I}+d_{2} \Phi+\left[\mathbf{L}(\mathbf{p}, \mathbf{q})-\Gamma^{-}(\mathbf{p}, \mathbf{q}, \mathbf{B})\right] \frac{\ell}{2 i \omega},
$$

where $\mathbf{L}(\mathbf{p}, \mathbf{q})=2(\mathbf{p} \cdot \mathbf{q}) \mathbf{I}-\mathbf{p q}-\mathbf{q p}$ and $\Gamma^{-}(\mathbf{p}, \mathbf{q}, \mathbf{B})$ are tensors of rank two, linear in $\mathbf{q}$, that determine the anisotropy in diffuse scattering. Without a magnetic field it is well known that $\Gamma^{0}(\mathbf{p}, \mathbf{q})=2(\mathbf{p} \cdot \mathbf{q}) /(1-\langle\cos \theta\rangle)$. When a magnetic field is present, the first order correction in the magnetic field is separated as $\Gamma^{-}(\mathbf{p}, \mathbf{q}, \mathbf{B})=\Gamma^{0}(\mathbf{p}, \mathbf{q})+B \delta \Gamma^{-}(\mathbf{p}, \mathbf{q}, \hat{\mathbf{B}})$. Because of the symmetry relation of Eq. (凷), one has $\Gamma^{-}(\mathbf{p}, \mathbf{q}, \mathbf{B})=\Gamma^{-}(\mathbf{p}, \mathbf{q},-\mathbf{B})^{*}$. This implies that $\delta \Gamma^{-}(\mathbf{p}, \mathbf{q}, \hat{\mathbf{B}})=-\delta \Gamma^{-}(\mathbf{p}, \mathbf{q}, \hat{\mathbf{B}})^{*}$, so that $\delta \Gamma^{-}(\mathbf{p}, \mathbf{q}, \hat{\mathbf{B}})$ must be anti-hermitian. Mirror symmetry imposes in addition that $\mathbf{T}_{\mathbf{p} \mathbf{p}^{\prime}}(\mathbf{B})=\mathbf{T}_{-\mathbf{p}-\mathbf{p}^{\prime}}(\mathbf{B})$ and thus $\Gamma^{-}(-\mathbf{p},-\mathbf{q}, \mathbf{B})=\Gamma^{-}(\mathbf{p}, \mathbf{q}, \mathbf{B})$. The general form of the tensor $\delta \Gamma^{+}(\mathbf{p}, \mathbf{q}, \hat{\mathbf{B}})$ allowed by symmetry has already been discussed in Ref. [11] and will be only slightly different for $\delta \Gamma^{-}$:

$$
\delta \Gamma_{i j}^{-}(\mathbf{p}, \mathbf{q}, \hat{\mathbf{B}})=i b_{1} \operatorname{det}(\mathbf{p}, \mathbf{q}, \hat{\mathbf{B}}) \delta_{i j}-i \varepsilon_{i j k} p_{k}\left[i b_{2}(\hat{\mathbf{B}} \cdot \mathbf{q})+i b_{3}(\hat{\mathbf{B}} \cdot \hat{\mathbf{p}})(\mathbf{p} \cdot \mathbf{q})\right]+i b_{4}\left[p_{k} \Phi_{k i} q_{j}+q_{i} p_{k} \Phi_{k j}\right]
$$

where the $b_{i}$ are real-valued coefficients to be determined.

The contribution of the first and the last scattering events is obtained by multiplying the tensor for the ladder diagrams of Eq. (16) by free Green tensors $\mathbf{G}_{0}$. This gives the incoherent contribution to the coherent backscattering,

$$
\begin{aligned}
\mathbf{G}_{0} \mathbf{G}_{0}^{*} \cdot L^{+}\left(\mathbf{p}, \mathbf{p}^{\prime}, \mathbf{q}\right) \cdot \mathbf{G}_{0} \mathbf{G}_{0}^{*} & \simeq \mathbf{G}_{0} \cdot d(\mathbf{p}, \mathbf{q}) \cdot \mathbf{G}_{0}^{*} \mathbf{G}_{0}^{*} \cdot d\left(-\mathbf{p}^{\prime},-\mathbf{q}\right) \cdot \mathbf{G}_{0} \\
& \simeq\left(1-i \ell^{*} \hat{\mathbf{p}} \cdot \mathbf{q}\right) \widetilde{\rho}\left(\mathbf{q}=0, z, z^{\prime}\right)\left(1+i \ell^{*} \hat{\mathbf{p}}^{\prime} \cdot \mathbf{q}\right) \Delta_{\hat{\mathbf{p}}} \Delta_{\hat{\mathbf{p}}^{\prime}} \\
& \simeq \widetilde{\rho}\left(\mathbf{q}=0, z-\ell^{*} \hat{p}_{z}, z^{\prime}+\ell^{*} \hat{p}_{z}^{\prime}\right) \Delta_{\hat{\mathbf{p}}} \Delta_{\hat{\mathbf{p}}^{\prime}},
\end{aligned}
$$

in terms of the Fourier transform of the ladder propagator $\widetilde{\rho}\left(\mathbf{q}, z, z^{\prime}\right)$, which is obtained from the solution of the diffusion equation (35). The coherent contribution depends on the most-crossed diagrams $C_{i j l k}^{+}$. The tensor $C_{i j l k}^{+}$is obtained from $L_{i j k l}^{-}$ after reversing the indices $k$ and $l$, according to Eq. (14), and after adding the contribution of the first and the last scattering event. In this calculation of the tensor $C_{i j l k}^{+}$, the reciprocity transformation (14) for the normal components $z$ and $z^{\prime}$ of the tensor has been neglected. The result is finally evaluated at reflection where $z=z^{\prime}=0$. The components of the ingoing and outgoing wave vectors $\mathbf{p}, \mathbf{p}^{\prime}$ perpendicular to the $z$ axis are denoted by $\mathbf{p}_{\perp}, \mathbf{p}_{\perp}^{\prime}$. Finally the coherent contribution can be written 


$$
\begin{aligned}
C_{i j l k}^{+}\left(\mathbf{p}, \mathbf{p}^{\prime}, \mathbf{p}+\mathbf{p}^{\prime}, \mathbf{B}\right) & =\widetilde{\rho}\left(\mathbf{p}_{\perp}+\mathbf{p}_{\perp}^{\prime},-\ell^{*} \hat{p}_{z}, \ell^{*} \hat{p}_{z}^{\prime}\right) \times \\
\Delta_{i l} \Delta_{j k} & +i\left[\Delta_{i l} M_{j k}^{-}\left(\frac{\mathbf{p}_{\perp}^{\prime}-\mathbf{p}_{\perp}}{2}, \mathbf{p}_{\perp}+\mathbf{p}_{\perp}^{\prime},-\mathbf{B}\right)+M_{i l}^{-}\left(\frac{\mathbf{p}_{\perp}-\mathbf{p}_{\perp}^{\prime}}{2}, \mathbf{p}_{\perp}+\mathbf{p}_{\perp}^{\prime}, \mathbf{B}\right) \Delta_{k j}\right]
\end{aligned}
$$

with the definition

$$
M_{i j}^{-}(\mathbf{p}, \mathbf{q}, \mathbf{B})=-\zeta \Phi_{i j}-\frac{\ell}{\omega} \Gamma_{i j}^{-}(\mathbf{p}, \mathbf{q}, \mathbf{B})
$$

and $\zeta=2\left(i d_{2}-\eta\right)$ is the Mie generalization of the parameter $F$ in Eq. (73) of Ref. 四, which was shown to produce a rotation of the polarization vector in the linear polarization channels of coherent backscattering. To first order in the magnetic field, the front factor $\widetilde{\rho}\left(\mathbf{p}_{\perp}+\mathbf{p}_{\perp}^{\prime},-\ell^{*} \hat{p}_{z}, \ell^{*} \hat{p}_{z}^{\prime}\right)$ of Eq. (44) is evaluated by replacing the parameter $L_{a}$, which was an absorption length for the evaluation of the light intensity in Eq. (35), by a factor depending on the backscattering angle according to:

$$
\frac{1}{L_{a}^{2}} \rightarrow\left(\mathbf{p}_{\perp}+\mathbf{p}_{\perp}^{\prime}\right)^{2}
$$

In second order in the magnetic field, $\lambda$ would be present here as well as stated in Eq. (16).

The values of the coefficients $b_{i}$ can only be found by solving a system of four coupled equations that one obtains when inserting Eqs. (42,43) into Eq. (19), and which is not reported explicitly here. The contribution of $b_{3}$ always vanishes in Eq. (44), since it is proportional to the scalar product $\mathbf{p} \cdot \mathbf{q}$ in Eq. (43), which is transformed into $\left(\hat{\mathbf{p}}^{\prime}-\hat{\mathbf{p}}\right) \cdot\left(\hat{\mathbf{p}}+\hat{\mathbf{p}}^{\prime}\right)=0$ in the operation involved in Eq. (14). For the same reason there is no contribution of $\Gamma^{0}(\mathbf{p}, \mathbf{q})$ in Eq. (44). Selection rules for the polarization are obtained in the helicity basis by considering the product

$$
C_{\sigma \sigma^{\prime}}^{+}=\overline{P_{i k}^{\sigma}}(\hat{\mathbf{p}}) C_{i j k l}^{+} P_{j l}^{\sigma^{\prime}}\left(\hat{\mathbf{p}}^{\prime}\right)
$$

In this calculation, the terms proportional to $\zeta$ in Eq. (45) disappear, as well as the contribution from $b_{4}$ which is longitudinal as can be seen from Eq. (43). Among the four terms of $\delta \Gamma(\mathbf{p}, \mathbf{q}, \hat{\mathbf{B}})^{-}$, only the terms proportional to $b_{1}$ and $b_{2}$ survive, and Eq. (37) is obtained for $C_{\sigma \sigma^{\prime}}^{+}$.

\section{References}

[1] E. Akkermans, P. E. Wolf, R. Maynard, and G. Maret. Theoretical study of coherent backscattering of light by disordered media. J. Phys. France, 49:77-98, 1988.

[2] A. A. Golubentsev. Suppression of interference effects in multiple scattering of light. JETP, 59(1):26-32, 1984.

[3] F. C. MacKintosh and S. John. Coherent backscattering of light in the presence of time-reversal-noninvariant and paritynonconserving media. Phys. Rev. B, 37(4):1884-1897, 1988.

[4] B. A. Van Tiggelen, R. Maynard, and T. M. Nieuwenhuizen. Theory for multiple light scattering from Rayleigh scatterers in magnetic fields. Phys. Rev. E, 53(3):2881-2908, 1996.

[5] A. S. Martinez and R. Maynard. Faraday effect and multiple scattering of light. Phys. Rev. B., 50(6):3714-3732, 1994.

[6] J. Q. Lu, A. A. Maradudin, and R. F. Wallis. Enhanced backscattering in a magnetic field. Waves in Random Media, 1:309-339, 1991.

[7] F. A. Erbacher, R. Lenke, and G. Maret. Multiple light scattering in magneto-optically active media. Europhys. Lett., 21(5):551-556, 1993. The value for the effective Verdet constant given on page 555 , and should be divided by a factor $\sqrt{2}$ due to a forgotten factor of 2 in Eq. (3).

[8] D. Lacoste, B. A. Van Tiggelen, G. L. J. A. Rikken, and A. Sparenberg. Optics of a Faraday-active Mie sphere. J. Opt. Soc. Am. A, 15:1636-1642, 1998.

[9] D. Lacoste and B. A. Van Tiggelen. Journal of Quantitative Spectroscopy and Radiative Transfer. JQSR, 63:305-19, 1999. 
[10] E. Akkermans, P. E. Wolf, R. Maynard, and G. Maret. Theoretical study of the coherent backscattering of light by disordered media. J. Phys. France, 49:77-98, 1988.

[11] D. Lacoste and B. A. Van Tiggelen. Transport mean free path for magneto-transverse light diffusion. Europhys. Lett., 45(6):721-725, 1999.

[12] A. Sparenberg, G. L. J. A. Rikken, and B. A. Van Tiggelen. Observation of photonic magneto-resistance. Phys. Rev. Lett., $79(4), 1997$.

[13] H. C. Van de Hulst. Light Scattering by Small Particles. Dover, New York, 1980.

[14] T. K. Xia, P. M. Hui, and D. Stroud. Theory of Faraday rotation in granular magnetic material. J. Appl. Phys., 67(6):273641, 1990.

[15] A. Ishimaru. Wave Propagation and Scattering in Random Media, volume 1. Academic Press, San Diego, 1978.

[16] R. Landauer. Barrier interaction time in tunneling. Rev. Mod. Phys., 66(1):217-228, 1994.

[17] A. Bott and W. Zdunkowski. Electromagnetic energy within dielectric spheres. J. Opt. Soc. Am. A, 4(8):1361-1365, 1987.

[18] P. Chýlek, J. T. Kiehl, and M. K. W. Ko. Optical levitation and partial-wave resonances. Phys. Rev. A, 18(5):2229-33, 1978.

[19] H. Y. Ling. Theoretical investigation of transmission through a Faraday-active Fabry-Perot etalon. J. Opt. Soc. Am. A, 11:754-758, 1994.

[20] M. B. Van der Mark, M. P. Van Albada, and A. Lagendijk. Light scattering in strongly scattering media: Multiple scattering and weak localization. Phys. Rev. B, 37(7):3575-3592, 1988.

[21] L. Landau, E. Lifchitz, and L. Pitayevski. Electrodynamics of Continuous Media. Mir, Moscow, 1967.

[22] G. L. J. A. Rikken and B. A. Van Tiggelen. Observation of magnetically induced transverse diffusion of light. Nature, 381:54-55, 1996.

[23] E. E. Gorodnichev, S. L. Dudarev, and D. B. Rogozkin. Coherent wave backscattering: Exact solution of the albedo problem. Phys. Lett. A, 144(1):48-54, 1990.

[24] R. Lenke and G. Maret. Private Communication. 


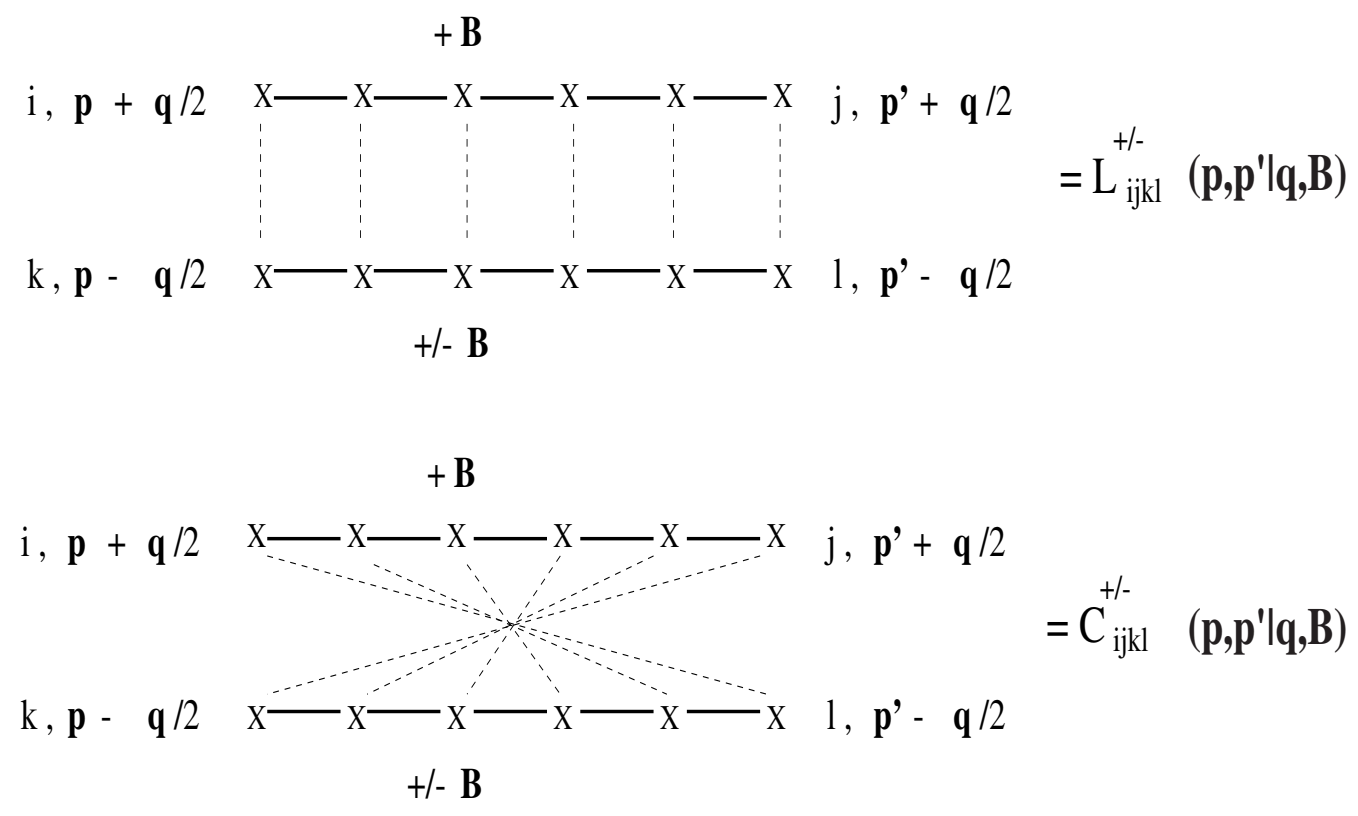

Figure 1: Ladder diagrams $L_{i j k l}^{ \pm}$and the most-crossed diagrams $C_{i j l k}^{ \pm}$in a magnetic field. Bold lines denote the Dyson-Green tensor, the crosses denote T-matrices and dotted lines connect identical particles. 
(a)

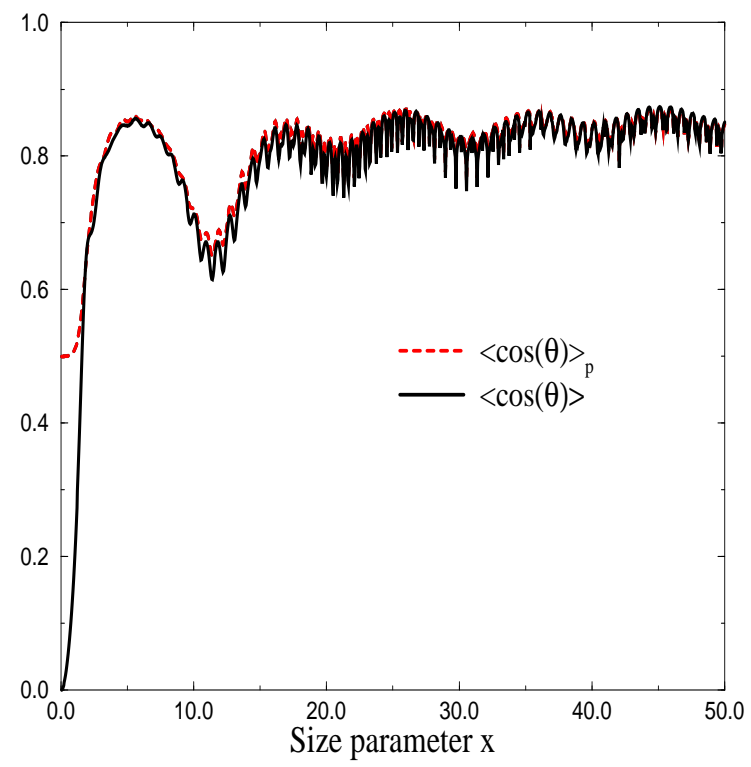

(b)

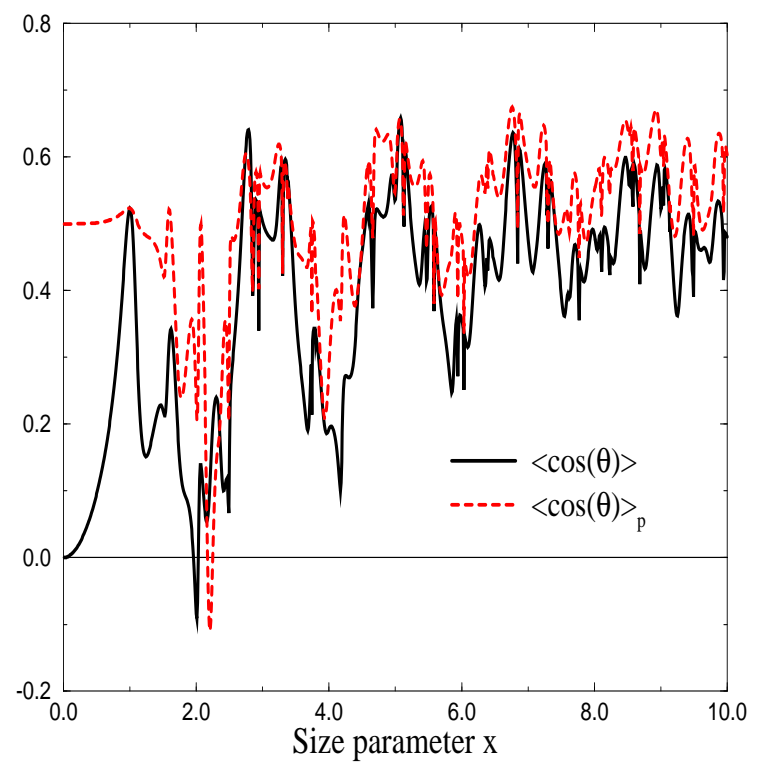

Figure 2: Asymmetry parameters $\langle\cos \theta\rangle$ and $\langle\cos \theta\rangle_{p}$ as a function of the size parameter $x$ for a relative index of refraction $m=1.33$ (a) and $m=2.73(\mathrm{~b})$.

(a)

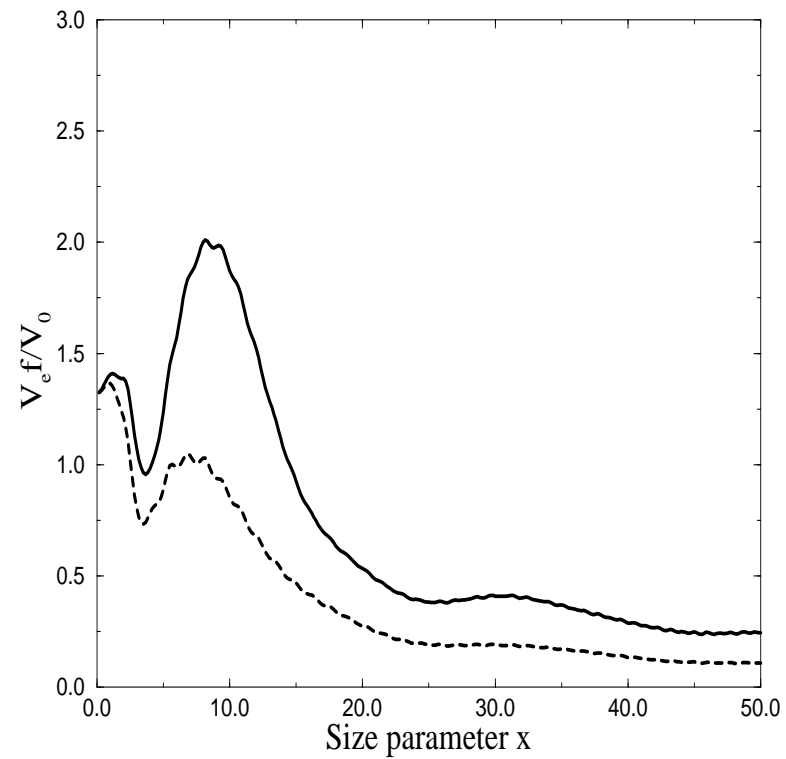

(b)

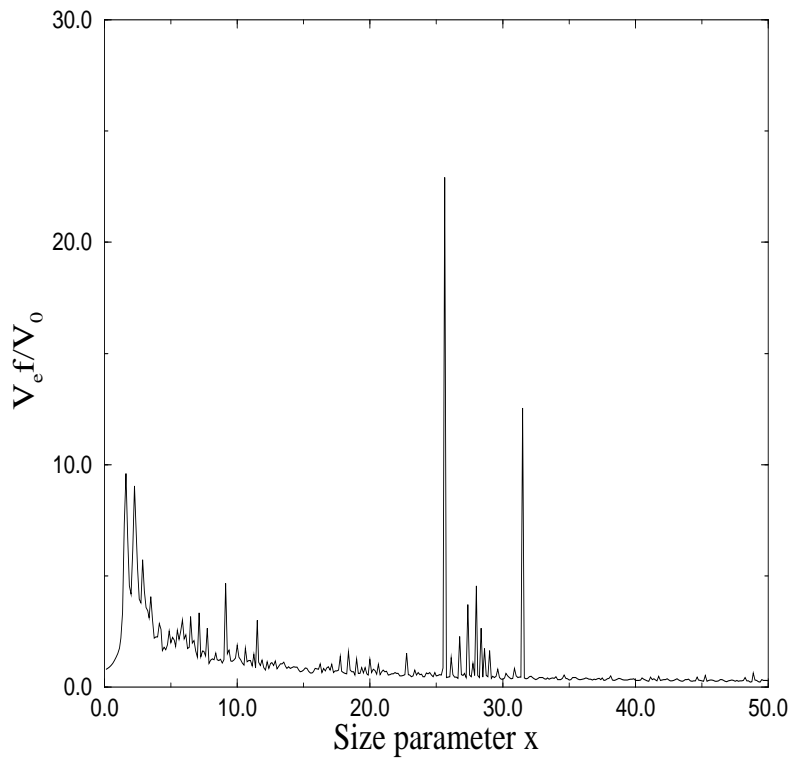

Figure 3: Plot of the effective Verdet constant $\frac{V_{\text {eff }}}{f V_{0}}$ as a function of the size parameter $x$. (a) The solid line represents the solution of Eqs. (20-23), containing contributions from all the rays incident on the sphere, whereas the dashed line corresponds to the contribution of only the first partial wave $J=1$ (the ray with central impact in geometrical optics). The scattering medium has an index of refraction of 1.7, the value in the experiment of Erbacher et al.. (b) The same plot for an index of refraction of 2.73 for which resonances are clearly visible. The host medium is in both cases glycerol (of index of refraction 1.47). A general decrease of the effective Verdet constant for increasing size parameter $x$ can be observed in both plots. 
(a)

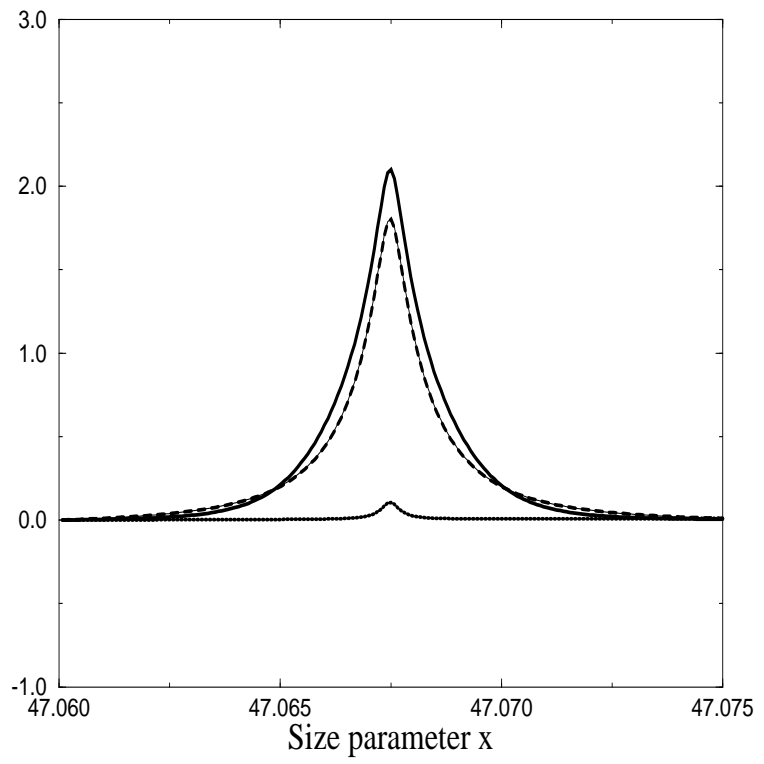

(b)

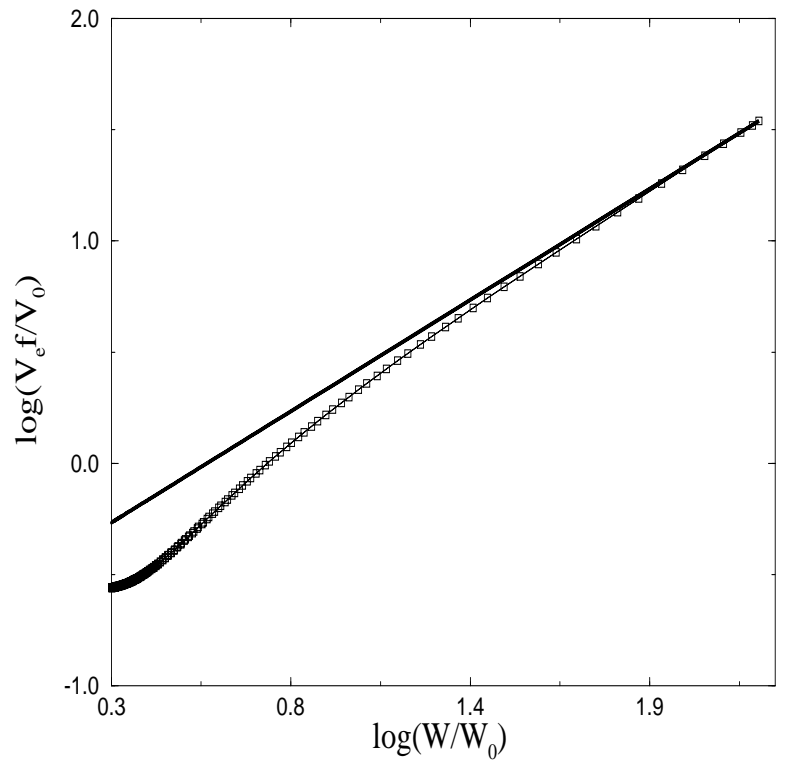

Figure 4: Near a particular resonance curve of a water sphere of index $m=1.334-1.5 \cdot 10^{-9} i$ the following curves have been plotted: (a) Respectively from the upper part of the figure to the bottom: a plot of $\log \left(V_{e f f} /\left(f V_{0}\right)\right)(\operatorname{solid}), \log W$ (dashed), and the scattering cross-section $Q_{\text {scatt }}$ (dotted) as a function of the size parameter $x$. (b) Close to this particular resonance, $\log \left(V_{\text {eff }} /\left(f V_{0}\right)\right)$ is shown against the total electromagnetic energy $W$ (dots), to be compared with a line of slope one (solid), the prediction of Eq. (34). 


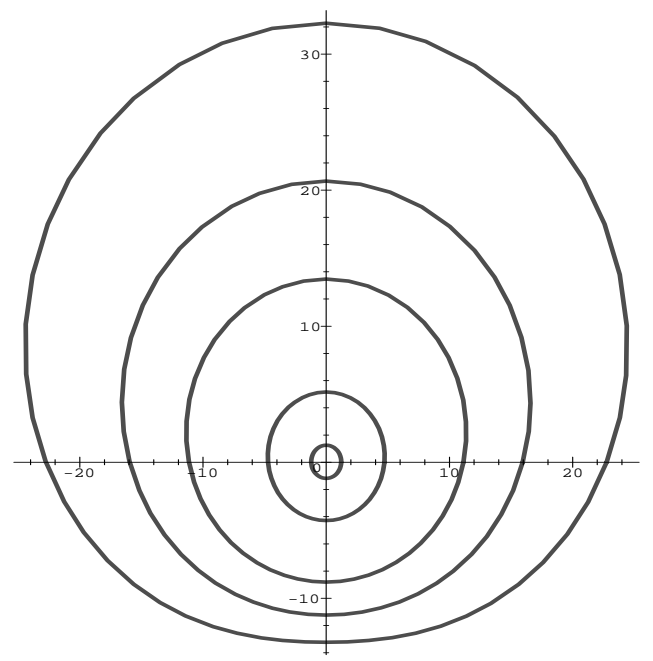

Figure 5: Polar plot of the lines of constant enhancement factor of the coherent backscattering cone in a magnetic field, in the helicity-conserving channel, for $b_{1}=1.8 \cdot 10^{-2}$. The magnetic field is along the horizontal axis on this graph. The graduations of the axis represent the dimensionless parameter $\mu=\omega \ell \theta$. For instance, a graduation of 10 corresponds to an angle of $145 \mathrm{mrad}$ (for the mean free path $\ell^{*} \simeq 90 \mu \mathrm{m}$, and the wavelength $\lambda=457 \mathrm{~nm}$ mentioned in the text). Without magnetic field or for a magnetic field perpendicular to the slab, these lines of constant enhancement factor would have been circles. 